\title{
LA PORTERÍA DEL COLEGIO MÁXIMO DE LA COMPAÑÍA (CÓRDOBA, ARGENTINA). LOS RECURSOS PARA SU INTERPRETACIÓN
}

\author{
Josefina Piana y Melina Malandrino ${ }^{1}$
}

\begin{abstract}
Resumen
El objeto del presente trabajo es la "portería" del Colegio Máximo de la Compañía de Jesús en Córdoba; este espacio, ubicado en la base de la torre Sur de la Iglesia, era el único ingreso al Colegio y al Noviciado de la Orden. El objetivo es identificar funciones y técnicas constructivas del periodo jesuítico. A pesar de las diversas actividades albergadas desde 1767, ese sector edilicio no ha sufrido intervenciones significativas desde el siglo XVII. Y aún en estos casos, se ha accedido a los registros escritos pertinentes. En 2012, la UNC restituye este ámbito a la Compañía de Jesús y comienzan las tareas de investigación y recuperación. En la primera etapa se recopiló y analizó la documentación escrita y gráfica (siglos XVII al XX); la segunda consistió en la aplicación de los protocolos propios de la arqueología histórica para contrastar la información. Aquí se analizan los resultados, conjuntamente con las posibilidades que brinda el espacio para la interpretación del sitio.
\end{abstract}

Palabras clave: Jesuitas, paraquaria, Colegio Máximo, recuperación.

\begin{abstract}
The purpose of this paper is the porter's lodge of the Maximum College of the Society of Jesus in Córdoba; this room is placed in the base of the south tower of the Church, it was the only entrance to the Order's College and Novitiate. The objective is to identify the function and the building techniques of the jesuit period. In spite of the diverse activities being hold there since 1767, this section has not suffered any meaningful interventions since the XVII century. And yet in this case, it was possible to access to the corresponding written registers. In 2012, the UNC returned this place to the Society of Jesus and the investigation and recovery task began. In the first stage, the graphic and written documentation was gathered and analyzed (from XVII till XX century); the second stage consisted in the application of the typical protocols of the historical archaeology to contrast the information. In this paper, the results were analyzed, jointly with the possibilities that this place provides for site interpretation.
\end{abstract}

Keywords: Jesuits, paraquaria, Maximum College, recovery.

\footnotetext{
${ }^{1}$ Universidad Católica de Córdoba. josefinapiana@yahoo.com.ar; melimalandrino@outlook.com
} 


\section{Introducción}

El presente trabajo forma parte un proyecto más amplio, que aborda la temática jesuítica en el territorio de la antigua Provincia de la Paraquaria. El mismo está radicado en el Centro de Investigación e Interpretación de la Paraquaria y el Instituto de Patrimonio "Marina Waisman" de la Universidad Católica de Córdoba. El método de abordaje está basado en la interdisciplina entre las especialidades de los investigadores del equipo, contrastando fuentes históricas (éditas e inéditas) con los testimonios arquitectónicos existentes. Los objetivos generales son proveer investigaciones de base para intervenciones de restauración e interpretación del patrimonio.

\section{La "Portería" en el conjunto de las propiedades urbanas de la Compañía}

Desde su instalación en el año 1599, hasta la expulsión en 1767, en los terrenos que la Compañía de Jesús poseía en el asiento urbano de Córdoba, funcionaron diferentes instituciones de la Orden: Noviciado, Colegio Máximo, Templo de San Ignacio, Procuración de Provincia, Botica, Real Colegio Convictorio de Nuestra Señora de Monserrat, Casa de Beatas, Seminario San Francisco Javier y Noviciado viejo. Del conjunto de propiedades, que ocuparon más de cuatro manzanas, se centrará la mirada en un pequeño ámbito, ubicado en la base de la torre Sur del Templo Mayor con acceso desde el atrio, donde se ubicaba la "portería" del Colegio Máximo, único ingreso al Colegio y al Noviciado de la Orden. (Piana y Malandrino, 2010).

\section{El sentido histórico de la "Portería"}

Si bien es un espacio arquitectónico de pequeñas dimensiones, su valor simbólico siempre es muy alto, y asume distintos significados según las funciones, los ministerios y los tiempos históricos. Hasta el momento de la expulsión de la Orden, es la única puerta de ingreso al Colegio Máximo, al Noviciado y a las oficinas del Prepósito Provincial. Este ingreso se realiza a través del atrio del Templo, lo que manifiesta el indisoluble vínculo entre la educación y la religión. (Figura 1)

Para los novicios, es el paso para "abandonar el siglo". En tanto al ámbito del Noviciado solo se accede a través de los claustros del Colegio, esta puerta de ingreso significa el abandono de la vida civil para cumplir con el bienio de probación.

Para los Padres y Hermanos de la Orden, "los nuestros" como se los designa en los documentos de la época, es el punto central de la recepción de los misioneros de Ultramar. Estos llegan al Colegio Máximo, donde permanecen un tiempo más o menos largo para aprender las lenguas generales necesarias para sus ministerios, o bien para completar los estudios de Gramática, Filosofía y Teología, exigidos por las Constituciones de la Orden.

En tanto el Colegio Máximo está facultado para otorgar grados universitarios desde 1622, los estudiantes externos que residen en el Real Colegio Convicto- 
rio de Nuestra Señora de Monserrate, ingresan diariamente por este espacio al "edificio de letras", donde asisten a las lecciones y cumplimentan los exámenes exigidos.

El 12 de julio de 1767, es la puerta por la que ingresa el sargento Fernando Fabro y su tropa, para ejecutar la Pragmática Sanción de Carlos III; también es la puerta por la que se inicia el destierro de los miembros de la Compañía de Jesús (Peramás, 2008).
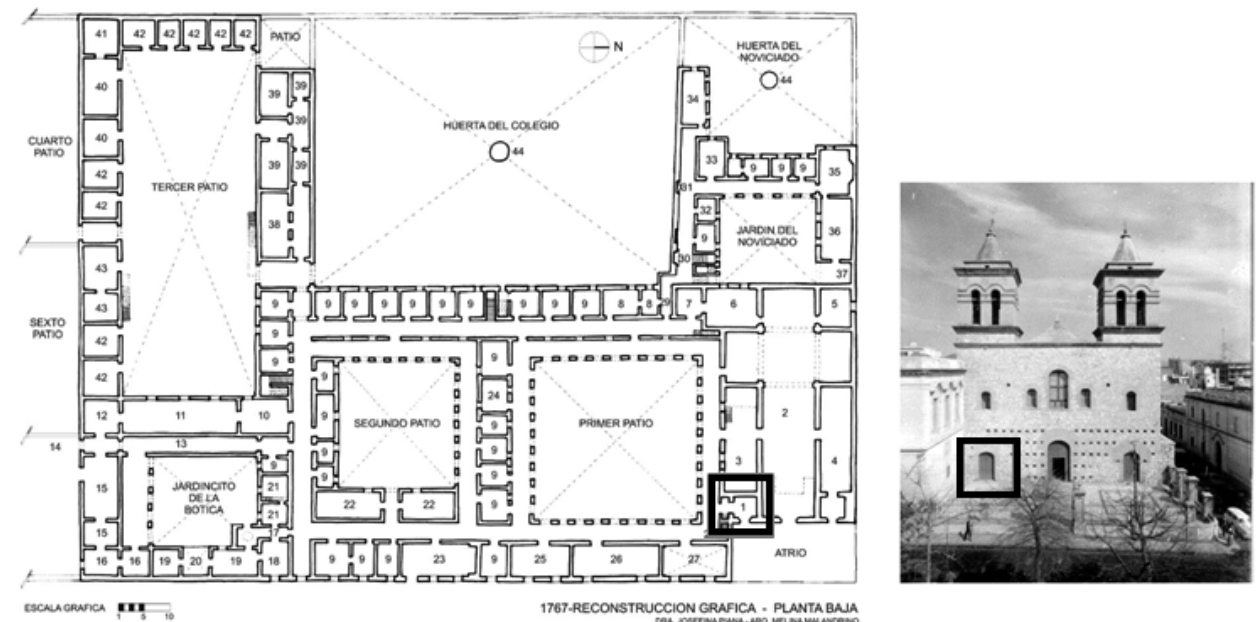

Figura 1: Plano del Colegio Máximo en 1767 (reconstrucción Piana-Malandrino) y fotografía de la fachada de la Iglesia de la Compañía de Jesús; en ambas se señala la portería.

\section{Después de la expulsión: los avatares de la Portería}

Luego de la expulsión, desde 1767 y hasta 1808, el edificio es administrado por la Orden Franciscana. En ese lapso se clausura el ingreso desde el atrio y se abre una nueva entrada a la Universidad por la actual calle Obispo Trejo. A partir de este momento solo se tiene acceso al espacio de la portería desde el claustro; esto conlleva a la pérdida su función original, y comienza a ser utilizado como depósito.

En el año 1859, cuando la Compañía de Jesús se restablece en Córdoba, el gobierno provincial le restituye el antiguo noviciado para residencia y el Templo Mayor. El reintegro no incluye la base de la torre Sur y el actual Salón de Grados (antes Capilla de españoles o de Congregantes), lo que conlleva la anulación de la escalera de acceso al coro de la Iglesia desde el claustro.

Desde mediados del siglo XIX y hasta el año de 2012, las administraciones de la Universidad, tanto en su etapa provincial como nacional, utilizan la antigua portería como depósito de insumos de limpieza y cafetería de los empleados; y a fines de la década del '90, la Federación Universitaria de Córdoba (FUC) construye un entrepiso metálico. 
Al cabo de 245 años, y después de arduas y prolongadas gestiones, en diciembre de 2012 la Universidad Nacional de Córdoba restituye a la Compañía de Jesús la base de la torre Sur, la antigua "portería” del Colegio Máximo. Los antecedentes de esta acción se remontan al año 1944, cuando la Comisión Nacional de Museos, Monumentos y Lugares Históricos (CNMMLH), mediante Resolución No871/44, expresaba:

"El Superior de la Compañía de Jesús, Rvdo. P. Pedro Paravano, hace fundada exposición tendiente a obtener la integridad del gran Monumento Histórico que es la Compañía de Jesús, de Córdoba.

Los miembros de la Sub-Comisión de Monumentos y Lugares Históricos, que suscriben, considera que corresponde gestionar ante la Intervención de la Universidad de Córdoba el canje de los locales (...) Con ello se obtendría, no solo una perfecta delimitación de ambas propiedades en toda su altura, suprimiendo la interferencia actual, sino que se devolvería a los locales mencionados el destino para el que fueron construidos, o sea, acceso directo al coro por la torre y Capilla de Españoles" (CNMMLH, 1944).

\section{Una investigación previa: la información de los documentos históricos}

Paralelamente a las gestiones administrativas para la restitución, se lleva adelante la investigación histórica. A esos efectos, son consultados el Archivo Histórico de la Universidad Nacional de Córdoba, del Centro de Documentación de Arquitectura Pública (CEDIAP), el archivo de la Comisión Nacional de Museos, Monumentos y Lugares Históricos y el Archivo Histórico de la Provincia de Córdoba. Los documentos gráficos y manuscritos de los siglos XVII al XX, permiten comprobar que el espacio conservaba una integridad histórica en condiciones de ser recuperada.

Los documentos gráficos consultados fueron (Figura 2):

- Plano del Colegio Máximo del Hermano Antonio Forcada (ca. 1748) (Furlong y Buschiazzo, 1943).

- Plano de la Universidad Nacional de Córdoba realizado por el Ingeniero A. S. Gonzalez, año 1893 (Archivo UNC).

- Plano de la Compañía de Jesús en Córdoba del Arquitecto C. Onetto, año 1940 (CEDIAP).

- Reconstrucción virtual de los edificios centrales de la Compañía de Jesús en Córdoba (Piana y Malandrino, 2010).

Los documentos escritos consultados proceden de la Junta de Temporalidades:

- Testimonio de los inventarios de los bienes ocupados a los regulares de la Compañía del Nombre de Jesús, año 1771 (Archivo UNC).

- Entrega que hace el Sargento Mayor don Fernando Fabro, al Sota Síndico de la Universidad, año 1773 (Archivo UNC). 

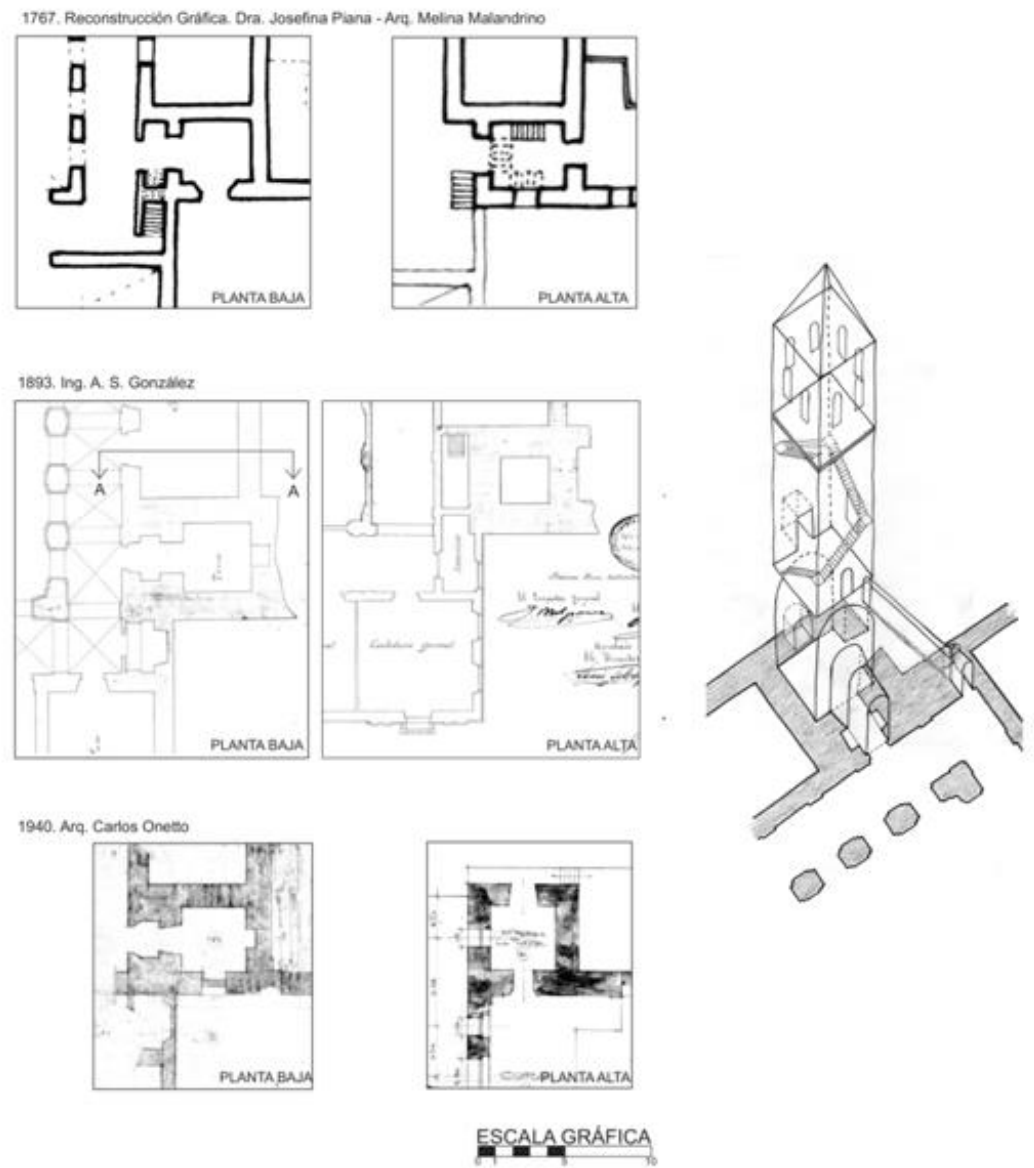

Figura 2: Izquierda, la portería en tres etapas históricas: 1767

(Piana-Malandrino), 1893 (Ing. Gonzalez), y 1940 (Arq. Onetto). Derecha: esquema geométrico del espacio de los 3 niveles de la torre, portería, acceso al coro y campanario.

De este último documento se extrae la descripción más detallada de la portería y su relación con el resto de las construcciones:

“- Seguida a la pieza que antecede, se halla otra en arranques sin construir, del mismo material, con dos rejas de hierro a la calle a la parte del naciente, sin puertas ni ventanas.

- Siguese a la pieza antecedente una escala por la que se sube a las torres de la Iglesia de este Colegio, las que se reconocieron tener la una, una campana grande con otras tres medianas, la una de éstas quebrada o raja$d a$, sin lengüeta, como así mismo otras dos, que sirven la una [folio 44v.] para repetir las horas del reloj y la otra para los cuartos; y la fábrica del reloj muy maltratado y descompuesto; y la otra torre sin campana alguna. 
- Habiendo bajado de dichas torres a la prosecución de la entrega del claustro del principal claustro y primer patio, se reconoció que debajo de la escalera referida antecedente, se halla un cuartito más pequeño de la misma construcción, con puerta de una mano con cerradura y llave, faltando el postiguito a dicha puerta.

- Seguido a la pieza que antecede, se halla la portería principal, con dos puertas con cerraduras y una llave, que hace a ambas, y la que cae al pretil, con sus clavos grandes de bronce; y entre ambas puertas una alacenita con puertas de dos manos, sin cerradura ni llave y un cuadro grande, redondo viejo, con su marco que contiene la Gloria y el Infierno.

- Se sigue la Capilla de Congregantes [...]”" (UNC, 1773).

\section{El proyecto de contrastación histórica}

Concretada la restitución de la antigua portería a la Compañía de Jesús, y debido a la condición de Monumento Histórico Nacional y Patrimonio de la $\mathrm{Hu}$ manidad del sitio, se solicita a la CNMMLH la visita de personal técnico para definir los lineamientos del proyecto de recuperación y puesta en valor.

Siguiendo las indicaciones de la Comisión Nacional, en junio 2013 se realizan tareas de liberación de las intervenciones del siglo XX: cañerías de agua, cableada de sistema eléctrico y entrepiso. Se efectúan dos cateos sobre la pared Norte de la base de la torre Sur de la Iglesia de la Compañía de Jesús.

- El primer cateo cubre una superficie de $15 \mathrm{~cm}$. X $40 \mathrm{~cm}$. a $2.50 \mathrm{~m}$. sobre el nivel actual de piso. El mismo indica un revoque realizado a comienzos del siglo XX; es probable que el mismo se extienda por toda la superficie interna de la base de la torre hasta la altura en que se inicia la bóveda, lo que se visualiza con claridad con los efectos de luz rasante. Sobre ese revoque tardío aparecen varias manos de pintura al agua, de colores ocres y celestes verdosos. En algunos puntos del cateo, debajo del revoque tardío aparece el revoque original de la superficie. Es de advertir que las denominaciones de "revoque tardío" y "original" se fundamentan en el tipo de material que los componen.

- El segundo cateo se realiza sobre el fondo de la hornacina que se encuentra en la pared Norte de la base de Torre. En este caso, la humedad había separado el revoque de la pared de ladrillo original, por lo que el cateo no pudo respetar las medidas previstas.

- Se detectan las diferentes manos de pintura, revoque tardío, marmolina (propio del periodo jesuítico para los espacios de culto o de relevancia) y revoque original.

En agosto 2014 se eleva a la CNMMLH el "Informe tareas de prospección realizadas durante el año 2014", en el que se consigna: 
"Teniendo como objetivo la liberación de los elementos construidos desde mediados del siglo XX en la base de la torre sur, en tanto estos no permitían o distorsionaban la correcta lectura del espacio, se procedió a:

Apertura del acceso a la torre desde el atrio de la iglesia, lo que implicó la demolición del muro de ladrillos (levantado 15 años antes de la fecha), que cegaba la abertura desde el interior y extracción de las puertas del placar construido en el vano. (Figura 3-1) Quedó de esta manera liberada la portada de ingreso que había sido restaurada por el Arq. Carlos Onetto en la década de 1940 y que fuera el ingreso original al Colegio Máximo desde el atrio de la iglesia. Resulta interesante señalar que en los depósitos de la Compañía de Jesús se encontró la antigua llave original de la portada, que es la que se utiliza actualmente para proceder a su apertura desde el interior de la base de la torre.

Remoción del entrepiso metálico y su escalera de acceso para recuperar la altura original y la lectura de la bóveda. Para la liberación de esta intervención realizada hace alrededor de 10 años, y a fin de no comprometer la estabilidad estructural de los muros, se cortaron al ras los perfiles tipo IPN. (Figura 3-2)

Remoción de la mesada de cocina con todas sus instalaciones de gas y agua, elementos que habían sido incorporados también hace alrededor de diez años. (Figura 3-3)

En el tránsito de acceso desde la antigua portería al claustro de la Universidad, se liberó el cielorraso suspendido y los espacios de guardado generados sobre los muros, intervenciones de una antigüedad no mayor a los quince años. Su liberación deja a la vista la bóveda que soporta la escalera original de acceso al coro de la iglesia. (Figura 3-4)

La Universidad Nacional de Córdoba reubicó los tableros eléctricos que controlaban la iluminación del Salón de Grados, que se encontraban alojados en uno de los placares del tránsito antes mencionado.

Se realizaron prospecciones para identificar el solado original, escalones y hornacina de la pared Norte donde originalmente se encontraba una cruz de madera sobre su peana" (Figura 3-5; 6 y 7) (Piana, 2014:2).

\section{Consideraciones finales: puesta en valor para su interpretación}

Los objetivos de la recuperación edilicia de la base de la torre Sur son la restauración de las portadas de ingreso desde el atrio y de acabados superficiales de la antigua portería, y recuperación de la escalera de ingreso al coro.

El ámbito de la antigua portería se presenta como un espacio de excelentes características para organizar en él una muestra interpretativa que permita explicar la funcionalidad y los valores simbólicos del lugar durante el período jesuítico, concluyendo con el relato del Padre Peramás sobre aquella noche de 1767: 
"Animado, pues, Fabro con el razonamiento llegó finalmente al Colegio y hoy, en el 12 Domingo, y puestas las centinelas por afuera y la ranchería (así llaman la casa de los esclavos), él con el resto de la tropa, con la bayoneta calada y con orden de hacer fuego a la menor resistencia, se vino a la portería, a donde se estuvo a las 3. A esta hora, estando el cielo nublado, que parecía tener sentimiento de nuestra desgracia, tocan la campanilla de la portería y llaman el P. José Paez para una confesión; avisó el portero al P. Rector Pedro Juan Andreu, quien le señaló compañero: acudieron los dos, y al abrir el portero, he aquí que entran en tropel y Fabro pidiendo paso franco le puso dos pistolas a los pechos al pobre portero. Pregunto: ¿para qué fue esta precipitación y aparato de armas? ¿Acaso para resistir a 112 sujetos, la mayor parte jóvenes, que, noticiosos de lo que pasaba, saldrían a impedir la entrada? No por cierto; pues todos muy descuidados estaban en sus lechos entregados al descanso. Pues ¿para qué fue? Para resistir a solo tres sujetos, de los cuales dos pasaban de 60 años. $Y$ para tan pocos y tan flacos ¿tanto aparato? Oh! Qué tímido es el corazón humano, cuando en lo que emprende no lo acompaña la razón ni la justicia!” (Peramás, 2008: 22).

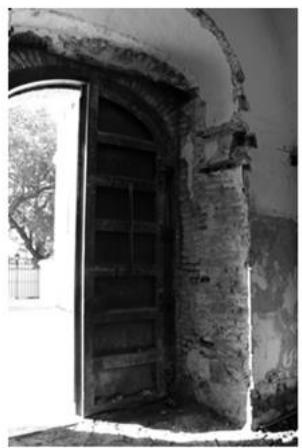

FOTO 1

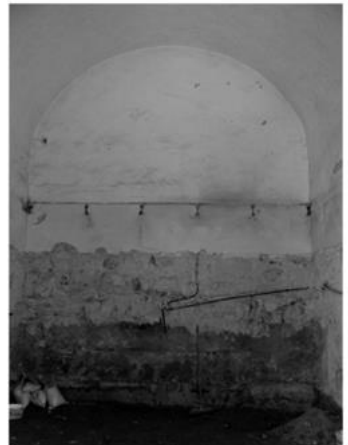

FOTO 2

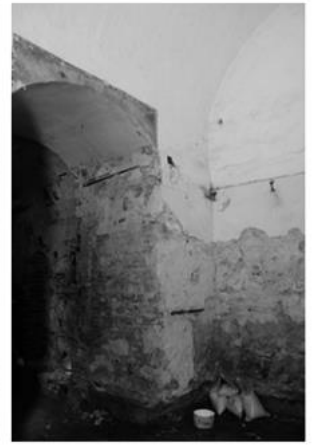

FOTO 3

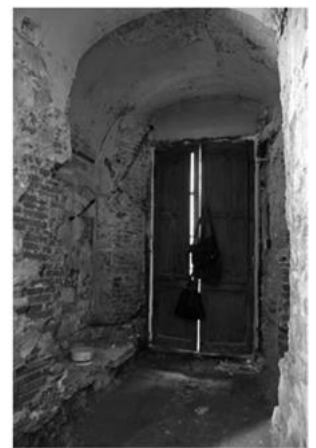

FOTO 4

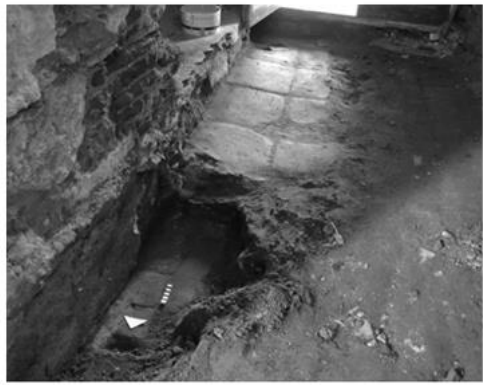

FOTO 5

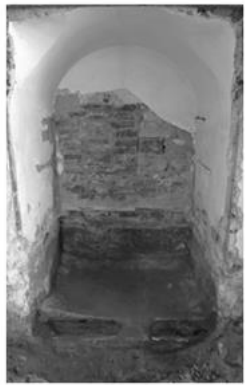

FOTO 6

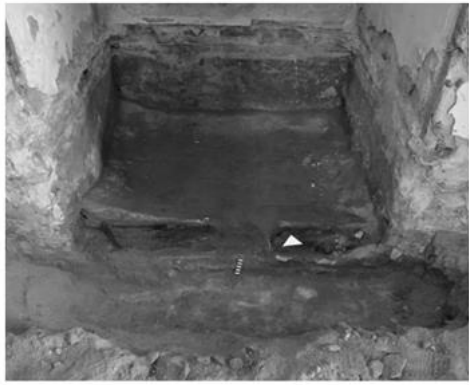

FOTO 7

Figura 3: Fotografias del "Informe tareas de prospección", numeradas aquí de 1 a 7. 


\section{Referencias bibliográficas}

ARCHIVO UNC. Caja 6, nro. 17, legajo 7. Testimonio de los inventarios de los bienes ocupados a los regulares de la Compañía del Nombre de Jesús, con los ornadenamientos conferidos por el excelentísimo señor don Francisco Bucareli al señor Comisionado don Fernando Fabro, quien procedió a ellos según se demuestra. Año de 1771.

ARCHIVO UNC. Legajo 3, No 15. Caja $\mathrm{N}^{\circ}$ 16. Entrega que hace el Sargento Mayor don Fernando Fabro, al Sota Síndico de la Universidad, de orden de los Señores de esta Municipal Junta, de la Capilla y alhajas del Noviciado que en ella existían, como asimismo las del Colegio Máximo, con la razón dada por dicho señor de las entregadas a la Iglesia Catedral y San Francisco, de mandado de esta Junta. Año de 1773 .

CNMMLH. Resolución No871/44. 17/10/1944.

FURLONG, Guillermo y BUSCHIAZZO, Mario. "Arquitectura Religiosa Colonial". En: Archivum I, Julio Diciembre de 1943, Cuaderno 2. Buenos Aires: Junta de Historia Eclesiástica Argentina.

GONZALEZ, Alejandro S. 1893. Planos de la Universidad Nacional de Córdoba. Archivo de la UNC.

ONETTO, Carlos. 1940. Relevamiento de la Compañía de Jesús, Córdoba. Archivo CEDIAP.

PERAMAS, José Manuel. 2008. Diario del destierro. Córdoba: EDUCC.

PIANA, Josefina y MALANDRINO, Melina. 2010. 1767. Colegio Máximo, Templo de San Ignacio, Noviciado, Botica y Procuración de Provincia de la Compañía de Jesús en Córdoba. Una reconstrucción edilicia según fuentes históricas. Córdoba: Compañía de Jesús.

PIANA, Josefina. 2014. Informe tareas de prospección realizadas durante el año 2014. 12/08/2014. Archivo CNMMLH.

Recibido: 13 de abril 2016.

Aceptado: 23 de septiembre 2016. 